\title{
O USO dO REIKI NA ASSISTÊNCIA À SAÚdE E NO SISTEMA ÚNICO DE SAÚdE
}

\section{The use of reiki in Health Care and the in the Unified Health System}

\author{
Sérgio Spezzia ${ }^{1}$, Solange Spezzia ${ }^{2}$ \\ 1. Cirurgião Dentista. Especialista em Gestão Pública pela Universidade Tecnológica Federal do Paraná. \\ Especialista em Gestão em Saúde pela Universidade Federal de São Paulo (UNIFESP) e Mestre em \\ Ciências pela Escola Paulista de Medicina - UNIFESP. ORCID: http://orcid.org/0000-0001-5622-5581. \\ 2. Terapeuta Holística e Reikiana. ORCID: http://orcid.org/0000-0003-3827-8780, \\ CONTATO: Rua Silva Bueno, 1001 | São Paulo | São Paulo | CEP 04208-050 | E-mail: sergio.
} spezzia@unifesp.br

COMO CITAR Spezzia S, Spezzia S. O uso do Reiki na assistência à saúde e no Sistema Único de Saúde. R. Saúde Públ. 2018 Jul.:1(1):108-115

COPYRIGHT Esta obra é disponibilizada nos termos da Licença Creative Commons - 4. 0 Internacional. É permitida a reprodução parcial ou total desta obra, desde que citada a fonte.

RESUMO O objetivo deste artigo foi investigar como procede a prática do Reiki e averiguar como é feito o atendimento com o Reiki no Sistema Único de Saúde (SUS). Foi realizada revisão bibliográfica, envolvendo estudos sobre como procede a prática do Reiki, incluindo os atendimentos que são feitos com essa técnica no SUS. A pesquisa foi feita nas bases de dados: LILACS e Google Acadêmico. Em 60\% dos estudos consultados, verificou-se que nos últimos 07 anos o uso do Reiki tem crescido entre médicos, enfermeiros, cirurgiões dentistas e que outros profissionais de saúde aplicam a técnica em hospitais, unidades de emergência, unidades psiquiátricas, salas de cirurgia e nos cuidados paliativos. Concluiu-se que, sob o enfoque dos princípios básicos preconizados pelo SUS que abrangem a prevenção e a integralidade dos atendimentos, o Reiki contribui para melhor aproveitamento dos recursos públicos e para melhoria na qualidade de vida dos pacientes.

PALAVRAS-CHAVE: Terapias Complementares. Sistema Único de Saúde. Integralidade em Saúde. 
ABSTRACT The purpose of this article was to investigate how the practice of Reiki proceeds and to investigate how Reiki care is done in the Unified Health System (SUS). A bibliographic review was carried out, involving studies on how Reiki practice proceeds, including the consultations that are done with this technique in the SUS. The research was done in the databases: LILACS and Google Scholar. In 60\% of the studies consulted, it has been verified that in the last 7 years the use of Reiki has grown among doctors, nurses, dentists and other health professionals apply the technique in hospitals, emergency units, psychiatric units, operating rooms and in palliative care. It was concluded that, under the emphasis of the basic principles recommended by the SUS that cover prevention and integral care, Reiki contributes to a better use of public resources and to improve the quality of life of patients.

KEYWORDS: Complementary Therapies. Unified Health System. Integrality in Health.

\section{INTRODUÇÃO}

E

$m$ conformidade com a Organização Mundial da Saúde (OMS), Terapias Alternativas, Complementares e/ou Integrativas são designadas como prática não alopática de assistência à saúde. Nelas buscase a prática holística do atendimento, fundamento que se baseia no vínculo forte firmado entre os pacientes e os profissionais de saúde praticantes. Essas terapias englobam o reiki, a meditação, a acupuntura, a terapia com florais, a naturopatia, a fitoterapia, dentre outras ${ }^{1,2}$.

Segundo estimativa da OMS (2002)2, o emprego dessas terapias em países desenvolvidos e em vias de desenvolvimento havia aumentado bastante. A OMS por intermédio de documento intitulado "Estratégia da OMS sobre Medicina Tradicional 2002-2005", almeja incrementar essas práticas, concomitantemente buscando que elas sejam empreendidas racionalmente com qualidade, segurança e eficácia.

As terapias alternativas podem ser agrupadas em terapias físicas; hidroterapia; fitoterapia; nutrição; ondas, radiações e vibrações; terapia de exercícios individuais e terapias mentais e espirituais. As terapias mentais e espirituais incluem o reiki, a meditação, o toque terapêutico, o relaxamento psicomuscular, a cromoterapia, entre outras ${ }^{3}$.
No Brasil, as práticas integrativas e complementares em saúde (PIC) ${ }^{4}$ são denominadas pelo Ministério da Saúde como Medicina Tradicional Alternativa e Complementar (MAC). As MAC não são consideradas integrantes das práticas de Medicina Convencional. Fazendo-se uso das PIC torna-se viável tratar os indivíduos como um todo, na causa central dos males apresentados e não se visa apenas e tão somente abranger os sintomas ${ }^{5}$.

As PIC visam acolher o sujeito, valorizando sua singularidade e subjetividade, por meio de uma postura autoeducativa que visa o desenvolvimento do potencial humano, buscando incentivo ao autoconhecimento, autocuidado e autotransformação. Essa prática possui alguns benefícios como baixo custo e eficácia ${ }^{6}$. Sabese que, nesse contexto, os conceitos de eficácia, eficiência e efetividade são extremamente importantes quando da elaboração de políticas públicas de saúde. A eficácia é uma medida que visa alcance de resultados, ela se preocupa com os fins e almeja alcançar os objetivos, enquanto que a eficiência é uma medida da utilização dos recursos, ela representa uma relação entre custos e benefícios e está voltada para a melhor maneira ou método pelo qual as coisas devem ser feitas ou executadas, com a finalidade de que os recursos 
sejam disponibilizados da maneira mais racional possível. Nela inexiste preocupação com os fins, ocorre preocupação apenas com os meios? Outro conceito que está correlacionado é a efetividade, nela a preocupação principal consiste em verificar a real necessidade e oportunidade para aplicação de medidas pelo governo, deixando claro que alguns setores serão beneficiados, enquanto outros não. A efetividade agrega os aspectos positivos que existem na eficiência e na eficácia ${ }^{8.9}$.

O Reiki ou "energia vital universal", dentre as PIC, constitui método preventivo, natural de cura, que almeja propiciar equilíbrio e harmonia da saúde física, mental, energética, emocional e espiritual. Atualmente o Reiki encontra-se bastante difundido no mundo, por propiciar recursos de autocuidado e autodesenvolvimento que podem auxiliar bastante os indivíduos 6 .

O objetivo deste artigo foi investigar como procede a prática do Reiki e averiguar como é feito o atendimento com a terapia Reiki no Sistema Único de Saúde.

\section{METODOLOGIA}

Foi realizado estudo de revisão narrativa da literatura, envolvendo artigos, trabalhos e estudos sobre como procede a prática do Reiki, incluindo os atendimentos que são feitos com essa técnica no SUS.

A pesquisa foi realizada nas bases de dados eletrônicas: Literatura Latino-Americana e do Caribe em Ciências da Saúde (LILACS) e Google Acadêmico. No LILACS empregou-se a expressão de busca: reiki and práticas integrativas complementares e obteve-se 16 registros. No Google Acadêmico utilizou-se a expressão de busca: reiki and sistema único de saúde and português and inglês and 2017 and 2016 and 2015 and 2014 and 2013 and 2012 and 2011 e obteve-se 36 resultados para consulta. Na base LILACS dos 16 estudos encontrados, 01 foi excluído por não possuir conteúdo concernente com o pesquisado; 01 foi excluído por possuir texto em espanhol e 09 foram excluídos por terem sido publicados em data anterior à delimitada para busca nos critérios de inclusão. Foram incluídos, portanto, 05 estudos. Na base Google Acadêmico, dos 36 registros encontrados, excluiu-se 10 por não possuírem conteúdo satisfatório para o desenvolvimento da pesquisa e 02 por estarem em outro idioma. Foram incluidos 24 estudos.

Apontamentos de livros, monografias, dissertações e teses sobre essa mesma temática também foram considerados e incluidos no artigo.

Os critérios de inclusão adotados levaram em consideração, estudos e artigos publicados nos idiomas inglês e português, nos últimos sete anos que versavam sobre o tema.

Excluíram-se artigos que não tinham conteúdo concernente com o selecionado para pesquisa, que eram de outros idiomas e que tinham sido publicados em data anterior à delimitada para busca.

\section{REVISÃO DE LITERATURA CARACTERISTICAS GERAIS DO REIKI E DE SUA PRÁTICA}

O Reiki veio ao Ocidente trazido por Hawayo Takata, que havia conhecido a técnica no Japão em 19350. Essa técnica apesar de ser oriunda do Japão agrega inúmeros adeptos em território brasileiro, possuindo aceitabilidade, tanto nos serviços públicos como nos privados ${ }^{11}$. 0 significado da palavra Reiki advém de duas palavras japonesas, o Rei, que significa Universal e o Ki, que significa energia vital'10,12,13.

O emprego da técnica ocorre por intermédio da imposição das mãos do aplicador reikiano sobre os pacientes, havendo dessa forma a estabilização dos canais de energia e o equilíbrio energético do organismo, propiciando resolução dos problemas de saúde ${ }^{14}$. Utiliza-se técnica de harmonização energética pela imposição das mãos em determinadas regiões ou locais do corpo, o que é capaz de restabelecer o equilíbrio da energia circulante. Nela uma pessoa transmite energia vital 
universal para outra, utilizando as mãos, no intuito de promover cura, potencializar a força vital e de agir equilibrando energias do corpo. A energia de cura segue para o corpo do paciente, advinda do ato de imposição das mãos, que age promovendo a autocura 12,13.15-17. A energia é transmitida em vários níveis simultaneamente, no nível físico, mental, emocional e energético ${ }^{18}$.

A aplicação do Reiki pode ser realizada em qualquer pessoa, sejam elas crianças, adultos ou idosos e comumente ocorre em centros energéticos do corpo denominados de chakras, os quais têm ligação com as glândulas, órgãos, vísceras e com o Sistema Nervoso. A palavra chakra significa roda no vocabulário sânscrito. Os sete chakras principais estão alinhados no sentido vertical da base do tronco ao topo da cabeça. Cada chakra possui uma cor e tem relação com a manutenção das funções físicas e emocionais, estando eles conectados a determinada glândula endócrina ou plexo nervoso. Os chakras absorvem energia universal primária, segmentam-na e enviam-na pelos nadis (rios de energia) ao sistema nervoso, sangue e glândulas endócrinas, visando alimentar o corpo ${ }^{10}$. Têm-se grandes e pequenos chakras ao longo do corpo, o primeiro, que é chamado chakra da raiz está localizado próximo ao cóccix; o segundo, chakra sacral ou esplênico, acha-se postado abaixo do umbigo ou próximo do baço; o terceiro, do plexo solar, localiza-se no abdômen em sua parte superior, embaixo do esterno; o quarto, chamado de chakra do coração, acha-se situado na parte média do esterno, sobre o coração ou timo; o quinto, chakra da garganta, está situado no pescoço, vizinho ao pomo de adão, sobre a laringe e tireoide: o sexto, da testa, denominado de anja, está postado na parte média da fronte, acima do nariz e o sétimo acha-se situado na porção do alto da cabeça. Todos os chakras devem funcionar perfeitamente, caso um deles cesse suas atribuições teremos problemas na absorção de energia, acarretando no não fornecimento de energia a determinadas células e órgãos do corpo. A terapia pelos chakras possibilita alimentar o corpo com energia, proporcionando bem-estar ao paciente, indo além do corpo físico ${ }^{19}$.
Os níveis de Reiki são nível I, II e III. No nível I. que é denominado o despertar, o indivíduo é apresentado ao contexto histórico da terapia, recebendo um símbolo, o $\mathrm{CHO} K U \mathrm{REI}$, que pode agir e ativar energia. No nível II, denominado de a transformação, ensina-se os indivíduos a transmitir e preparar a transmissão do Reiki à distância, nessa fase recebe-se mais dois símbolos de Reiki, o SEI HE KU e o HON SHA ZE SHO NEN. No nível III, ocorre uma divisão em dois níveis IIIA e IIIB. No nível IIIA é denominado mestre de si e o reikiano recebe conhecimentos acerca de como demonstrar flexibilidade frente às intempéries do cotidiano e recebe símbolos de Reiki idênticos aos dos níveis anteriores. No nível IIIB, designado de mestre professor, o reikiano recebe ensinamentos sobre como promover a iniciação a novos reikianos, recebendo os mesmos símbolos de niveis anteriores, além dos símbolos empregados pelo mestre para realizar as iniciações, são eles: DAI KOO MIO, RACU e SERPENTE DE FOGO²0.

No decorrer da aplicação do Reiki, convém seguir alguns procedimentos para se obter bons resultados, e, para tal, paciente e profissional devem manter pernas, braços e dedos descruzados, no intuito de evitar cessação do fluxo de energia; o reikiano posiciona-se por detrás do paciente para dar início à sessão, nesse contexto, pode ser adequado usar incensos e música suave; a duração da sessão oscila entre 45 e 60 minutos e cada posição deve ser mantida pelo paciente de 3 a 5 minutos, além disso, deve-se iniciar o tratamento pela cabeça, obedecendo as posições convencionais, entretanto, no decorrer da sessão, caso haja necessidade, pode-se posicionar as mãos sobre outras áreas ${ }^{21,22}$.

$\mathrm{Na}$ habilitação para aplicar o Reiki, o praticante deve passar, portanto por uma iniciação, que ocorre em níveis, respeitando-se determinado espaçamento temporal para cada qual. Essa preparação deve ser feita por um mestre de Reiki, que se encontra gabaritado para ensino e iniciação. Sabe-se que no decorrer da iniciação é ministrada uma aula teórica e ocorre um ritual em que o aluno recebe uma harmonização ou sintonização 
que fornecerá meios para que ele possa captar a energia vital do universo $0^{10,13,23}$.

A aplicação da terapia, além de ser de baixo custo, não promove efeitos adversos e mostra-se segura, provavelmente devido ao fato, de que na atualidade não é necessário ocorrer licenciamento para atuar, exercendo a terapia Reiki ${ }^{12}$.

O National Center for Complementary and Alternative Medicine classificou o Reiki como modalidade de medicina energética e o Reiki foi oficialmente recomendado pelo National Health Service Trusts e pelo The Prince of Wales's Fondation for Integrated Health. Nesse contexto, e na modalidade de medicina energética pode-se fazer uso dele em instituições assistenciais, como hospitais e postos de saúde ${ }^{13}$.

O uso do Reiki tem crescido nos últimos sete anos entre médicos, enfermeiros, cirurgiões dentistas e outros profissionais de saúde, e a técnica tem sido aplicada em hospitais, unidades de emergência, unidades psiquiátricas, salas de cirurgia e dentre os recursos usados nos cuidados paliativos. Na Literatura, entretanto, denota-se escassez de pesquisas ou estudos realizados em unidades básicas de saúde e na estratégia de saúde da família acerca do tema12,23.

\section{O REIKI NA ASSISTÊNCIA À SAÚDE PÚBLICA}

No ano de 2006, por meio da Portaria 971 de 03 de maio, o Ministério da Saúde, obedecendo à diretriz preconizada pela OMS, firmou a Política Nacional de Práticas Integrativas e Complementares no Sistema Único de Saúde (SUS), fornecendo para atendimento cinco procedimentos (homeopatia, medicina tradicional chinesa/acupuntura, plantas medicinais e fitoterapia, medicina antroposófica e termalismo social/crenoterapia) ${ }^{24.25}$. O ato viabilizou o atendimento com uso dessas práticas por profissionais de saúde do SUS na Atenção Primária, tanto nas Unidades Básicas de Saúde (UBS), como nas Equipes de Saúde da Família. Essa política visa promover, prevenir e recuperar a saúde, através da utilização de métodos baseados no acolhimento e em relações de vínculo terapêutico entre usuário, família e profissionais envolvidos, almejando proceder a orientações acerca do autocuidado com a saúde, educando também os pacientes?.

Nesse contexto, a Atenção Primária à Saúde (APS) detém papel de destaque, uma vez que reúne características peculiares que permitem melhor entrosamento entre as partes contactantes no decorrer dos atendimentos. Deve haver troca de informações constantemente entre as partes para que ocorra uma comunicação eficaz, além disso, a humanização dos atendimentos também contribui para inter-relacionamento positivo entre os profissionais de saúde e os indivíduos que buscam assistência à saúde ${ }^{26}$. No SUS, ainda na Assistência à Saúde Básica ou Primária, as PIC, que devem ser coordenadas pela Estratégia de Saúde da Família, visam promover a integralidade nos atendimentos realizados ${ }^{27}$.

A Portaria n 849 de março de 2017, firmada pelo Ministério da Saúde, estabeleceu algumas outras terapias, que passaram a compor as Práticas Integrativas e Complementares em Saúde (PICS) no SUS, incorporando 14 novas atividades, totalizando 19 práticas para atendimento, considerando as 5 práticas já existentes desde 2006. As 19 terapias disponíveis para uso da população passaram a ser constituídas por ayurveda, homeopatia, medicina tradicional chinesa, medicina antroposófica, plantas medicinais/fitoterapia, arteterapia, biodança, dança circular, meditação, musicoterapia, naturopatia, osteopatia, quiropraxia, reflexoterapia, reiki, shantala, terapia comunitária integrativa, termalismo social/crenoterapia e yoga 24,25,28.

Nesse contexto, a partir de março de 2018 passaram a ser disponibilizadas mais 10 novas PICS para uso, são elas: apiterapia, aromaterapia, bioenergética, constelação familiar, cromoterapia, geoterapia, hipnoterapia, imposição de mãos, ozonioterapia e terapia de florais, totalizando 29 terapias 24,25 .

No âmbito do SUS, essas práticas complementares têm mostrado aceitação dos pacientes, que são os que buscam esses atendimentos e têm interesse por eles ${ }^{29}$. 
$\mathrm{Na}$ atualidade, $30 \%$ dos municípios pertencentes aos 27 estados e Distrito Federal dispõem de PICS na assistência à saúde, e existem 1.708 municípios brasileiros que possuem atendimento voltado para as PICS, havendo concentração de 78\% dos serviços na Atenção Básica, que é considerada Porta de Entrada do SUS; 18\% na Atenção Especializada ou Secundária e 4\% na Atenção Terciária ou Hospitalar. Estimase que cerca de 7.700 estabelecimentos fornecem PICS à população, configurando $28 \%$ das UBS com atendimento ${ }^{30}$.

\section{DISCUSSÃO}

Segundo Cunha, (2011)31, o Reiki possibilita estabelecimento de equilíbrio entre o excesso ou a falta de vitalidade, o que é feito por profissional capacitado por intermédio do toque das mãos, do olhar e/ou do sopro em locais específicos do corpo dos pacientes, que apresentam bloqueios de energia. $\mathrm{O}$ objetivo pretendido é fortalecer o corpo do paciente, permitindo a liberação de toxinas causadoras de desequilíbrio, visando ainda propiciar estado de consciência favorável e vida plena.

Em suma, a terapia promove tratamento holístico, trata causas e sintomas das patologias, fortalecendo o sistema imunológico, aliviando a sintomatologia dolorosa e liberando as toxinas ${ }^{31}$. Convémenfatizar queas PICS, segundo denominação realizada pela OMS, configuram práticas que detêm custo diminuto frente à demanda de atendimentos de várias doenças, atuando coadjuvantemente aos tratamentos principais preconizados ${ }^{2}$.

Alguns estudos verificaram que a prática do reiki em casos de estresse, ansiedade, fadiga e sedação mostrou-se positiva e foi capaz de atenuar quadros dolorosos presentes ${ }^{12,32}$. Estudo realizado por Oliveira $(2014)^{13}$, denotou que o Reiki possui perfil favorável nas intervenções terapêuticas realizadas para quadros de estresse, melhorando inclusive a qualidade de vida. Evidenciou-se também que são necessários mais estudos de natureza multidisciplinar para tornar possivel a compreensão dos possíveis mecanismos psicofisiológicos e físicos que estão envolvidos com o reiki.

Outro estudo afirma que existe interesse crescente pelas terapias complementares no âmbito do SUS. Profissionais de saúde da Atenção Primária apoiam seu uso, além disso, sabe-se que existe demanda elevada por atendimentos com essas terapias ${ }^{33}$.

De acordo com Andrade \& Costa (2010) ${ }^{34}$, no Brasil o campo das PICs vem ascendendo e essas práticas têm sido disseminadas em igrejas, clínicas particulares, entidades não governamentais (ONGs), bem como em instituições públicas de saúde.

É importante que se proceda a conscientização dos profissionais de saúde acerca de como as PICs podem ser utilizadas, evitando desconhecimento para sua aplicabilidade clínica ou para encaminhamento de casos para tratamento por outros profissionais capacitados. Nas graduações na área da saúde as PICs não fazem parte da grade curricular da maioria das instituições. Algumas vezes ocorre ministração de disciplina acerca do tema, no entanto, inexiste formação satisfatória²7.

A aplicação do reiki permite a humanização do cuidado, uma vez obedecidos princípios de acolhimento, escuta, confiança, avaliação e possivel encaminhamento adequado a outros profissionais de saúde quando necessário, visando resolução da demanda apresentada pelos pacientes ${ }^{35}$.

O custo-benefício da terapia reiki no SUS pode mostrar-se vantajoso. $\mathrm{O}$ baixo custo somado à grande abrangência populacional são atrativos que levam à escolha dessa prática. Pode-se optar por utilizar essa terapia em doenças epidêmicas, estas que normalmente despendem quantidade grande de recursos públicos para tratamento e que com o emprego dessas terapias tendem a economizar recursos dos serviços públicos de saúde ${ }^{36}$.

A técnica tem caráter preventivo e age na causa dos problemas, demonstrando desfecho satisfatório ao ser utilizada em casos de ansiedade, depressão, insônia, medo, pânico, nas situações com sintomatologia dolorosa presente e em outras situações que possuem indicação para uso ${ }^{37,38}$. 
O reiki pode ser aplicado em inúmeras doenças, tais como: sinusite, asma, fadiga, artrite, insônia, depressão, entre outras. Em pacientes cancerosos submetidos à quimioterapia e que desenvolvem efeitos adversos ou secundários, como a mucosite oral, por exemplo, a terapia reiki auxilia a restabelecer o bem-estar, reduzindo o estresse emocional, que é causado pelo câncer ${ }^{39-41}$.

No SUS somente em 2017 ocorreu a formalização dos atendimentos de reiki à população, o que representa um período pequeno em termos das adequações que sempre são necessárias para atendimento, no que tange principalmente ao pessoal capacitado e à estrutura física dos estabelecimentos de saúde, entretanto a médio e longo prazo os resultados da assistência realizada certamente mostrar-se-ão satisfatórios ${ }^{28}$.

Relacionado aos pacientes usuários da Atenção Básica anteriormente à regulamentação dos atendimentos de reiki foi possível detectar uma busca por terapias novas ou diferenciadas das que havia até então, englobando uma forma de tratamento que fosse feita sem o uso de medicamentos e que concomitantemente trouxesse sensação de bem-estar ${ }^{42}$.

São necessários mais estudos ou pesquisas científicas acerca das PICs, visando avaliar e incrementar a aceitabilidade social e institucional dessas práticas. Esses estudos devem envolver a aplicabilidade das PICs realizada também pelo SUS.

\section{CONCLUSÃO}

Concluiu-se que, sob o enfoque dos princípios básicos preconizados pelo SUS que abrangem a prevenção e a integralidade dos atendimentos, o Reiki contribui para melhor aproveitamento dos recursos públicos e para melhoria na qualidade de vida dos pacientes.

\section{REFERÊNCIAS}

1. Brasil. Ministério da Saúde. Portaria $n^{\circ} 971$, de 03 de maio de 2006. Aprova a Política Nacional de Práticas Integrativas e Complementares (PNPIC) no Sistema Único de Saúde. Brasília: Ministério da Saúde. 2006. Disponivel em:<http://dtr2001.saude. gov.br/sas/PORTARIAS/Port2006/GM/GM-971.htm>. Acesso em: 06 de janeiro de 2018.

2. Organização Mundial da Saúde. Estratégias da OMS sobre Medicina Tradicional 2002 - 2005. Disponivel em: <http:// whqlibdoc.who.int/hq/2002/WHO_EDM_TRM_2002.1_spa.pdf >. Acessado em 06 de janeiro de 2018.

3. Trovo MM, Silva MJP, Leão ER. Terapias alternativas/ complementares no ensino público e privado: análise do conhecimento dos acadêmicos de enfermagem. Rev Latin-Americ Enfermagem, 2003; 11(4):483-9.

4. Telesi Júnior E. Práticas integrativas e complementares em saúde, uma nova eficácia para o SUS. Rev Estudos Avançados, 2016; 30(86):99-112.

5. Scheffer M. Terapia floral do Dr. Bach: teoria e prática. 13. ed. São Paulo: Pensamento, 2011.

6. Ferrer VC. Reiki como uma Estratégia de Autocuidado e Promoção de Saúde Integral: uma realidade para o trabalhador do Distrito Federal. Monografia do Curso de Especialização em Saúde Mental, Álcool e Outras Drogas do Instituto de Psicologia da Universidade de Brasília. Brasília, DF, 2015

7. Chiavenato I. Recursos humanos na Empresa: pessoas, organizações e sistemas. 3.ed. São Paulo: Atlas, 1994. p. 67-76.

8. Torres MDF. Estado, democracia e administração pública no Brasil. Rio de Janeiro: Ed. FGV, 2004.

9. Spezzia S, Carvalheiro EM, Trindade LL. Uma análise das políticas públicas voltadas para os serviços de saúde bucal no Brasil. Rev Bras Odontol, 2015; 72(1/2): 109-13.

10. Mckenzie E. A Bíblia do Reiki: o guia definitivo para a arte do Reiki. São Paulo, 2010. 400p.

11. Brasil. Ministério da Saúde. Secretaria de Atenção à Saúde. Departamento de Atenção Básica. Política Nacional de Práticas Integrativas e Complementares no SUS: atitude de ampliação de acesso/Ministério da Saúde. 2. ed. Brasília : Ministério da Saúde, 2014. $96 p$.

12. Demir M. Can G. Celek E. Effect of Reiki on symptom management in oncology. Asian Pac J Cancer Prev, 2013; 14(8):4931-3.

13. Oliveira RMJ. Efeitos da prática do Reiki sobre aspectos psicofisiológicos e de qualidade de vida de idosos com sintomas de estresse: estudo placebo e randomizado. [Tese de Doutorado]. Escola Paulista de Medicina - Universidade Federal de São Paulo, 2013.

14. Diaz-Rodriguez L, Arroyo-Morales M, Cantarero-Villanueva I. Fernandez-Lao C, Polley M. Fernández-de-las-Peñas C. Uma sessão de Reiki em enfermeiras diagnosticadas com síndrome de Burnout tem efeitos benéficos sobre a concentração de $\lg \mathrm{A}$ salivar e a pressão arterial. Rev Latin-Americ Enfermagem, 2011; 19(5):1132-8.

15. Franco LC, Souza LAF, Pessoa APC, Pereira LV. Terapias não farmacológicas no alívio da dor neuropática diabética: uma revisão bibliográfica. Rev Acta Paulista de Enfermagem, 2011; 24(2):284-8.

16. Freitag VL, Dalmolin IS, Badke MR, Andrade A. Benefícios do Reiki em população idosa com dor crônica. Texto \& Contexto Enferm, 2014; 23(4):1032-40. 
17. Salles LF, Vannucci L, Salles A, Silva MJP. Efeito do Reiki na hipertensão arterial. Rev Acta Paul de Enferm, 2014; 27(5):479-84.

18. Usui M, Petter FA. Manual de Reiki do Dr. Mikao Usui. 6.ed. São Paulo: Pensamento, 2014. 79p.

19. Gerber R. Medicina Vibracional: uma medicina para o futuro. 9.ed. São Paulo: Cultrix, 2007. 463p.

20. Cordeiro LR, Souza AMA. Reiki com profissionais de saúde: iniciação de uma prática integrativa complementar para mudanças em níveis espiritual, profissional e pessoal. In: Matos K. SL de (Org.).Cultura de paz, ética e espiritualidade IV. Fortaleza: Edições UFC, 2013. p. 487-99

21. Eidt AC. O meio copo cheio - uma visão positiva que enlaça o Reiki e a psicologia corporal no cuidado da pessoa com câncer. In: Encontro Paranaense, Congresso Brasileiro de Psicoterapias Corporais, XVIII, XIII, Curitiba, 2013. Disponivel em: < http://www. centroreichiano.com.br/artigos/Anais 2013/EIDT,\%20Alyne\%20 Cavallari.\%200\%20meio\%20copo.pdf > Acessado em: 19 de outubro de 2016.

22. Garbin RF, Silva RS. Apostila, Reiki seminário nível 1, 2017.

23. Nogueira MVB, Lima AFA, Cordeiro LR, Santiago Júnior L, Costa ACS, Galdino DFS, et al. PRO-PET saúde UFC e terapia Reiki: relato de experiência. $11^{\circ}$. Congresso Internacional da Rede Unida, Anais. Rev Interface-Comunicação, Saúde, Educação, 2014 (Suplemento). Supl. 3.

24. Ministério da Saúde. Departamento de Atenção Básica. Mais 10 práticas integrativas são inseridas na PNPIC, 2018. Disponivel em: http://dab.saude.gov.br/portaldab/noticias. php?conteudo= \&cod=2872 Acessado em 17 de abril de 2018.

25. Brasil. Ministério da Saúde. Secretaria-Executiva. Secretaria de Atenção à Saúde. Glossário temático: práticas integrativas e complementares em saúde / Ministério da Saúde, SecretariaExecutiva, Secretaria de Atenção à Saúde. - Brasília: Ministério da Saúde, 2018. 180 p. Disponivel em: http://portalarquivos2. saude.gov.br/images/pdf/2018/marco/12/glossario-tematico.pdf Acessado em 17 de abril de 2018.

26. Coriolano-Marinus MWL, Andrade RS, Ruiz-Moreno L, Lima LS. Comunicação entre trabalhadores de saúde e usuários no cuidado à criança menor de dois anos no contexto de uma unidade de saúde da família. Interface-Comunicação, Saúde, Educação, 2015: 19(53):311-24.

27. Thiago SC. Medicinas e terapias complementares na visão de médicos e enfermeiros da Saúde da Família de Florianópolis. [Dissertação de Mestrado em Saúde Pública] - Universidade Federal de Santa Catarina, Florianópolis, 2009.

28. Brasil. Ministério da Saúde. Gabinete do Ministro. Portaria N ${ }^{0}$ 849, de 27 de março de 2017. Disponivel em: http://www.lex.com. br/legis_27357131_PORTARIA_N_849_DE_27_DE_MARCO_ DE_2017.aspx Acessado em 21 de fevereiro de 2018.

29. Freitag VL, Andrade A, Badke MR. O Reiki como forma terapêutica no cuidado á saúde: uma revisão narrativa da literatura. Rev Electr Trim de Enferm, 2015: 38:346-56.

30. Brasil. Ministério da Saúde. Portal do Departamento de Atenção Básica. Portaria Amplia Oferta de PICS. Publicação Acrescenta mais sete Novos Procedimentos no SUS, 2017. Disponivel em: http:// dab.saude.gov.br/portaldab/noticias.php?conteudo=_\&cod=2297 Acessado em 22 de fevereiro de 2018.

31. Cunha MTMC. Apostila Seminário de Reiki Tibetano, nível III. Brasília, 2011. 96 p.
32. Demir M, Can G, Kelam A. Effects of Distant Reiki On Pain Anxiety and Fatigue in Oncology Patients in Turkey: A Pilot Study. Asian Pac J Cancer Prev, 2015; 16 (12): 4859-62.

33. Sawni A, Thomas R. Pediatricians' attitudes, experience and referral patterns regarding complementary/alternative medicine: a national survey. BMC Complementary and Alternative Medicine. 2007; 7:18.

34. Andrade JT, Costa LFA. Medicina complementar no SUS Práticas Integrativas e Complementares sob a luz da Antropologia médica. Saúde \& Sociedade, 2010; 19(3):497- 508.

35. Chernicharo IM, Freitas FDS, Ferreira MA. Humanização no cuidado de enfermagem: contribuição ao debate sobre a Política Nacional de Humanização. Rev Bras Enferm, 2013; 66(4):564-70.

36. Sociedade Brasileira de Diabetes. Diretrizes da Sociedade Brasileira de Diabetes 2014-2015. In: Oliveira JEP, Vencio S. (Org.) São Paulo: AC Farmacêutica, 2015

37. Thrane S, Cohen SM. Effect of Reiki Therapy on Pain and Anxiety in Adults: An In Depth Literature Review of Randomized Trials with Effect Size Calculations. Pain Manag Nurs, 2014; 15(4): 897-908

38. Joyce J, Herbison GP. Reiki for depression and anxiety. Cochrane Database of Systematic Reviews, 2015; 4(CD006833). DOI: 10.1002/14651858.CD006833.pub2.

39. Granjeiro L. Reiki cada vez mais usado em doentes com cancro para reduzir efeitos da quimioterapia, 2013. Disponivel em: https:// lifestyle.sapo.pt/saude/noticias-saude/artigos/reiki-cada-vezmais-usado-em-doentes-com-cancro-para-reduzir-efeitos-daquimioterapia-2 Acesso em: 22 de fevereiro de 2018.

40. Orsak G, Stevens AM, Brufsky A, Kajumba M, Dougall AL. The Effects of Reiki Therapy and Companionship on Quality of Life Mood, and Symptom Distress During Chemotherapy. J EvidenceBased Complement Altern Med, 2015; 20(1) 20-7.

41. Rodrigues RB. Energia Vital Universal (Cura, equilíbrio, harmonia). Ed. Clube dos Autores, 2015. Disponivel em: < https:// books.google.com.br/books?id=CSeVCgAAQBAJ\&pg=PA4\&lpg=P A4\&dq=RODRIGUES,+2015+reiki\&source=bl\&ots=WqCuk4unBA \&sig=xrOYMncSJzP95AV $x H q H 9 p l \times O d O \& h l=p t B R \& s a=X \& v e d=0$ ahUKEwjbi_mXjPjTAhXEHJAKHQW7CzkQ6AEIIzAA\#v=onepage \&q=RODRIGUES\%2C\%2020 15\%20reiki\&f=false> Acesso em: 16 de outubro de 2016.

42. Souza IMC, Bodstein RCA, Tesser CD, Santos FAS, Hortale VA Práticas integrativas e complementares: oferta e produção de atendimentos no SUS e em municípios selecionados. Cad Saúde Pública, 2012; 28(11):2143-54. 